\title{
The mediating role of self-stigma and self- efficacy between intimate partner violence (IPV) victimization and depression among men who have sex with men in China
}

\author{
Liping Peng ${ }^{1 \dagger}$, Rui She ${ }^{2 \dagger}$, Jing $\mathrm{Gu}^{1,3}$, Chun Hao ${ }^{1,3}$, Fengsu Hou ${ }^{4}$, Dannuo Wei ${ }^{1}$ and Jinghua $\mathrm{Li}^{1,3^{*}}$
}

\begin{abstract}
Background: Previous studies have shown that intimate partner violence (IPV) is prevalent in men who have sex with men (MSM). Mental health problems among MSM with IPV victimization have become a growing concern. The present study examined homosexual self-stigma and self-efficacy as potential mediators of the association between IPV victimization and depression.

Methods: We recruited 578 MSM from 15 cities across China. Participants completed sociodemographic measures, the IPV-GBM (IPV among gay and bisexual men) scale, the Self-Stigma Scale-Short Form (SSS-S), the General SelfEfficacy (GSE) Scale and the Center for Epidemiologic Studies Depression 10 (CES-D-10). We calculated biascorrected 95\% confidence interval (Cl) for total, direct and indirect effects using bootstrapping to conduct mediation analyses.
\end{abstract}

Results: Findings showed that the prevalence of IPV victimization and depression were 32.7\% (189/578) and 36.0\% (208/578), respectively. Result from mediation analysis using structural equation modeling indicated that the association between level of IPV victimization and depression among MSM was fully mediated by higher homosexual self-stigma and lower self-efficacy. Homosexual self-stigma had a direct effect and an indirect effect via self-efficacy on depression.

Conclusion: The results provided evidence that integrated interventions that reduce self-stigma and foster selfefficacy could be promising approaches to decrease depression among MSM with IPV victimization.

Keywords: Men who have sex with men, Intimate partner violence, Depression, Self-stigma, Self-efficacy

\section{Introduction}

Intimate partner violence (IPV) is generally defined as physical, sexual, psychological or financial harm by a current or former intimate partners or spouse [1, 2], which is a significant public health issue among women. However, IPV is not exclusive to opposite-sex relationship. The emergency of research on men who have sex with men (MSM) has demonstrated that IPV occurs in

\footnotetext{
* Correspondence: lijinghua3@mail.sysu.edu.cn

${ }^{\dagger}$ Liping Peng and Rui She are co-first author.

'School of Public Health, Sun Yat-sen University, No.74, Zhongshan second road, Guangzhou, China

${ }^{3}$ Sun Yat-sen Global Health Institute, Sun Yat-sen University, Guangzhou, China

Full list of author information is available at the end of the article
}

male-male partnerships at rates similar to or higher than opposite-sex relationship [3]. A recent systematic review concluded that the estimated prevalence of lifetime IPV was $41.2 \%$ [95\% confidence interval (CI) 32.4-50.1\%] among MSM [3]. Studies in China also documented high prevalence of IPV among MSM: ranging from 18.7 to $51.0 \%$ for any form of IPV [4-7], 6.6-16.1\% for physical IPV, and $5.5-5.7 \%$ for sexual IPV. Some studies also show that IPV prevalence is higher in special groups of MSM, such as male sex workers (57.4\%) and men who have sex with men and women (37.6\%) [4, 7]. IPV experiences can result in a variety of high-risk sexual behaviors and health problems, such as substance use, engagement in unprotected anal intercourse, group sex,

(c) The Author(s). 2020 Open Access This article is distributed under the terms of the Creative Commons Attribution 4.0 International License (http://creativecommons.org/licenses/by/4.0/), which permits unrestricted use, distribution, and reproduction in any medium, provided you give appropriate credit to the original author(s) and the source, provide a link to the Creative Commons license, and indicate if changes were made. The Creative Commons Public Domain Dedication waiver (http://creativecommons.org/publicdomain/zero/1.0/) applies to the data made available in this article, unless otherwise stated. 
transactional sex, HIV infection [6, 7], depression, and suicide behaviors [3]. For instance, a survey among MSM in UK in the PROUD trial from 2012 to 2014 showed that clinically significant depressive symptom prevalence was approximately three times higher in MSM who reported IPV victimization (adjusted prevalence ratios $=2.57,95 \% \mathrm{CI}=1.71-3.86, P<0.001$ for lifetime IPV victimization; adjusted prevalence ratios $=2.93$, 95\% CI $=1.96-4.40, P<0.001$ for IPV victimization in last year) [8]. A survey among HIV-negative MSM in Northeastern China in 2014 showed that 18.7\% (89/476) of the participants reported being victims of any form of IPV (including physical, psychological and sexual) in the past 3 months and those who had been victims of IPV in the past 3 months were more likely to suffer from symptoms of depression (adjusted odds ratios $=2.8,95 \% \mathrm{CI}=$ 1.7-4.5, $P<0.05$ ) [6]. Although the issue of IPV and mental health has attracted more attention globally, researches on the relationship between IPV victimization and mental health among MSM in China are still rare. Thus, it is greatly warranted to have a closer look at the association between IPV and depression among MSM population in China.

Although the association between IPV and depression in MSM has been reported in literature, the potential pathways linking IPV and depression have been rarely explored. This is crucial for developing effective interventions to maintain and promote mental health of IPV victims. Lifetime IPV victimization among MSM was strongly associated with self-stigma against sexual minority status, which involves a process of incorporating negative societal views of homosexuality into the self-concept $[8,9]$. According to the minority stress theory $[10,11]$, sexual minority people in a heterosexual society are subjected to chronic stress related to their stigmatization, such as self-stigma and perceived stigma, which are prominent determinants of mental health for minority populations. Empirical evidence also suggested that selfstigma was significantly associated with greater risk of depression among Chinese MSM [12-14], especially among HIV-positive MSM. In addition, several studies have demonstrated that self-stigma was a significant mediator in the association between prejudiced experiences such as victimization and mental distress among sexual minorities $[15,16]$. This may suggest a possible mediation effect of self-stigma on the relationship between IPV victimization and depression in MSM [17].

Another potential mediator is self-efficacy, which denotes the belief in one's capabilities to organize and execute the courses of action required to manage prospective situations $[18,19]$. The perception of having personal control and confidence is one important factor in the study of contributors to mental health after exposure to potentially traumatizing events. Extant literature on self-efficacy in IPV victims were mainly focused on financial self-efficacy and condom negotiation self-efficacy among heterosexual women [20, 21]. Few studies have explored the impact of self-efficacy on the mental health of MSM with IPV victimization. The experience of IPV could be particularly harmful for self-worth and selfefficacy, due to the role that the interactions with others play in the development of self-representations [22]. A study conducted among Chinese MSM indicated that a higher level of general self-efficacy was associated with lower levels of depression [23]. General self-efficacy has been found to be a mediator between stressful life events and depressive symptoms among general population [24]. Thus, IPV may deteriorate self-efficacy, which in turn may increase the risk of depression. In addition, previous studies among sexual minority population revealed that self-stigma could weaken one's self-efficacy [25] and lead to negative health outcomes, such as unhealthy behavior [26] and adverse psychological problems (e.g., depression) [27].

Thus, the present study aimed to investigate the association between IPV victimization and depression among Chinese MSM, and to test the extent to which the association between IPV and depression would be mediated by self-stigma and self-efficacy. We hypothesized that: (1) IPV victimization would be associated with higher self-stigma and lower self-efficacy; (2) self-stigma would be negatively associated with self-efficacy and positively associated with depression; (3) self-efficacy would be negatively associated with risk of depression.

\section{Method}

\section{Study population and procedure}

A cross-sectional survey was conducted from April to June in 2019 in 15 cities across China, including five cities in East China (Sanya, Fuzhou, Hangzhou, Shenzhen and Qingdao), three cities in Midland China (Taiyuan, Changsha and Hefei), four cities in Northeast China (Changchun, Zhengzhou, Harbin and Urumqi), and three cities in West China (Lanzhou, Nanning and Kunming). The selected cities cover all main regions of China and are the main cities in their provinces. Because each city is the first-tier city or provincial capital city of China and their importance are of the same level, so the number of participants recruited by each city is approximately the same (40-50 per city), rather than sampling by population. Participants were recruited through local gay-friendly health consulting organizations and peer referrals. A professional questionnaire research platform (www.wjx.cn) was selected to release the online questionnaire. Participants were 
firstly briefed about the study purpose, procedure and benefits. Participants provided the informed consent and completed the online questionnaire by clicking to enter the website provided by fieldworkers. The online questionnaire took an average of 15 min to complete. Inclusion criteria were: 1) male aged 18 and above, 2) self-reported anal intercourse with at least one man in the last 6 months, and 3) has or has ever had an intimate partner. Fieldworkers checked the questionnaire upon completion and the questionnaire research platform would also review the logic errors of the questionnaire according to our preset logic rules, and screen out the invalid questionnaire. Each participant who had completed and submitted a qualified questionnaire was offered CNY 15 (about USD 2.5) as a compensation for their time spent on the survey. Of all the 1233 participants approached, 578 eligible participants with complete information were included in our survey, while 573 of them gave up filling midway or fell into the "trap item" which we designed in some of the scales to screen out invalid questionnaires with logic errors, and 82 of them fail to meet the inclusion criteria as reporting no intimate partner.

Ethics approval was obtained from the ethics committee of Sun Yat-sen University (Approval No. [2018] 049).

\section{Measurement}

\section{Background characteristic}

Sociodemographic information was collected including age, ethnicity (Han or other ethnicity), marital status (single, married, having girlfriend, having boyfriend, divorced, widowed, or others), education level (primary school or below, junior high school, senior high school, undergraduate, or postgraduate), personal monthly income, employment (full-time, part-time, student, unemployed, or retired), sexual orientation (homosexual, heterosexual, bisexual, or not sure), and history of sexually transmitted diseases (STDs).

\section{IPV victimization}

Five items with reference to IPV-GBM (IPV among gay and bisexual men) scale were developed to assess the IPV, which corresponded to five different domains: physical, sexual, monitoring, controlling and emotional IPV [28]. Example items to assess physical and sexual IPV are "Have any of your intimate partners ever tried to hurt you? This includes hitting you, punching you, kicking you, slapping you, pushing or shoving you, damaging your property, and other physical threats." and "Have any of your intimate partners ever force you to do something sexually that you didn't want to do? This includes forcing oral or anal intercourse, forcing you to have sex with someone else, refusing to wear a condom during sex when you requested to use, or any other sexual behavior that makes you feel uncomfortable." Response to each item is categorized as Yes or No (Yes is coded as 1 , and No is coded as 0 ). The variable of "level of IPV victimization" was a continuous variable, which added up the score of all five items, with a total mean score of 0.62 (standard deviations, $\mathrm{SD}=1.11$, range $=0$ $5)$ in this sample. The variable of "any IPV victimization" was defined as the presence of any of the investigated five types of IPV and was a dichotomous variable, which was coded as "1" for the participants who responded "Yes" to any one of the five items, and " 0 " for the participants who responded "No" to all five items. "Any IPV victimization" was used only in the descriptive analysis to calculate the prevalence of IPV victimization. In the correlation analysis and SEM analysis, we used "level of IPV victimization".

\section{Homosexual self-stigma}

The 9-item Self-Stigma Scale-Short Form (SSS-S) was used to measure the internalized stigma that was attributed to being homosexual self-stigma, which has been validated among Chinese sexual minority populations [29]. It consisted of three subscales: Cognitive (3 items), affective (3 items) and behavioral (3 items). An example item for cognitive subscale was "My identity as a gay is a burden to me." An example item for affective subscale was "I fear that others would know that I am a gay." An example item for behavioral subscale was "I avoid interacting with others because I am a gay." Reponses were rated using a 4-point Likert scale ranging from 1 (strongly disagree) to 4 (strongly agree). Average scores of all items were used, with a higher score indicating a stronger sense of self-stigma. The mean score of SSS-S in this sample was $1.94(\mathrm{SD}=0.59$, range $=1-4)$. The Cronbach's alpha was 0.91 in the present sample.

\section{Self-efficacy}

The General Self-Efficacy (GSE) Scale was used to assess self-efficacy. The GSE scale includes 10 items and has been adapted to Chinese MSM [30, 31]. Sample items include "Thanks to my resourcefulness, I can handle unforeseen situations." and "I can always manage to solve difficult problems if I try hard enough." Possible responses were $1=$ not at all true, $2=$ hardly true, $3=\bmod$ erately true, and $4=$ exactly true. Items were then summed to create a composite score for self-efficacy ranging from 10 to 40 , with higher score indicating higher level of self-efficacy. The Cronbach's alpha was 0.93 in the present sample.

\section{Depression}

The Center for Epidemiologic Studies Depression 10 (CESD-10) was used to measure depressive symptomatology by 
asking participants about their feelings of sadness and loneliness, difficulties in sleeping and concentrating, lost of interest and hope in the past week. The scale has been validated among Chinese MSM [32-34]. Symptom frequency was reported on a 4-point Likert-type scale ranging from never (0) to nearly every day (3). Each item score was summed up to generate the total score ranging from 0 to 30 and depression was taken as a continuous variable in the correlation analysis and structural equation modeling (SEM). The higher the score, the more severe the depression. But a cut-off of 10 was used to indicate the presence of probable depression [35]. The Cronbach's alpha was 0.89 in the present sample.

\section{Statistical analysis}

Data were exported directly from the online questionnaire system and analyzed using SPSS 25.0 (SPSS Inc., Chicago, IL). First, chi-square tests were used to investigate differences in prevalence of depression and IPV among participants with different demographic characteristics. Second, spearman's correlations were conducted to test the relationships between IPV, self-stigma, self-efficacy and depression. Third, structural equation modeling (SEM) was performed by AMOS 22.0 using maximum likelihood method to test the hypothesized mediation model. SEM requires a priori specification of both measurement and structural models. Confirmatory factor analysis (CFA) was firstly conducted to assess the goodness of fit of the measurement model for latent variables including homosexual self-stigma, selfefficacy, and depression. In the next step, the proposed SEM model was examined to test the mediation effects of self-stigma and self-efficacy in the association between IPV and depression. Background variables that were significantly associated with depression were controlled as covariates. To evaluate the overall model fit, we used indices including $x^{2 / d f}$ ratio, comparative fit index (CFI), incremental fit index (IFI), and root-mean-square error of approximation (RMSEA). For each index, the following criteria were applied: (1) $x^{2 / d f}$ ratio values less than 3 indicates a good model fit [36]; (2) CFI and IFI values greater than 0.9 indicates a good model fit [37]; and (3) for RMSEA, a value between 0.05 and 0.08 indicates an acceptable model fit [38]. Modification indices were inspected to discover the source of the lack of fit, and the model was adjusted accordingly. The total, direct, and indirect effects of the mediation model were estimated using bootstrapping, which has higher power than the commonly used Sobel test or causal steps approach [39]. Biascorrected $95 \%$ CI for each direct and indirect path were reported based on 5000 bootstrap samples. The level of statistically significance was set $p$ value $<0.05$ (two sided).

\section{Result \\ Background characteristics}

Among the 578 participants, 40.5\% $(n=234)$ were younger than 25 years old; the mean age was $28.6( \pm 7.2)$. Of those sampled, $90.8 \% \quad(n=528)$ were Han ethnicity; $54.3 \%(n=314)$ had attended university or above; $8.1 \%$ ( $n=47)$ were married with a woman; $15.7 \%(n=91)$ had had a monthly personal income of less than $1000 \mathrm{CNY}$ (160 USD); 66.1\% $(n=382)$ had full-time jobs; $81.3 \%$ $(n=470)$ had reported their sexual orientation as homosexual; $22.5 \% \quad(n=130)$ reported a history of STDs (Table 1).

\section{IPV prevalence}

$32.7 \%$ of the MSM participants reported at least one form of IPV victimization $(n=189)$. Emotional IPV was most frequently reported type (17.1\%), followed by 'monitoring' (15.1\%), 'sexual' (11.6\%), 'physical' (9.5\%), and 'controlling' (9.2\%). There was no statistically significant difference in terms of any IPV victimization prevalence rate between each group (Table 1).

\section{Depression prevalence}

Prevalence of probable depression was 36.0\% (208/578) in the present study. It varied with age and personal monthly income in this sample. Depression prevalence was the highest among those aged 18-25 years old (45.3\%), followed by 26-45 years old (30.1\%) and lowest among those aged above 45 years old (20.0\%). Depression prevalence was the highest among those had personal monthly income of 1001-3000 CNY (44.6\%), followed by $\leq 1000 \mathrm{CNY}$ (40.7\%), 3001-6000 CNY (33.6\%) and > $6000 \mathrm{CNY}(24.8 \%)$ (Table 1).

\section{Correlations between variables}

Means, standard deviations, and correlations between IPV, homosexual self-stigma, self-efficacy, and depression are presented in Table 2. The mean score for the three subscales of self-stigma was $2.13(\mathrm{SD}=0.70)$, $1.97(\mathrm{SD}=0.65)$, and $1.72(\mathrm{SD}=0.58)$, for affective, cognitive, and behavioral respectively. The mean score of general self-efficacy scale and depression scale (CES-D-10) was $27.45(\mathrm{SD}=6.00)$ and $7.55 \quad(\mathrm{SD}=$ $5.75)$, respectively. We found that IPV victimization was significantly positively correlated with depression $(\mathrm{r}=0.206, p<0.01)$. All the hypothesized mediating variables were significantly correlated with independent variables (IPV victimization) and dependent variables (depression) (Table 2). 


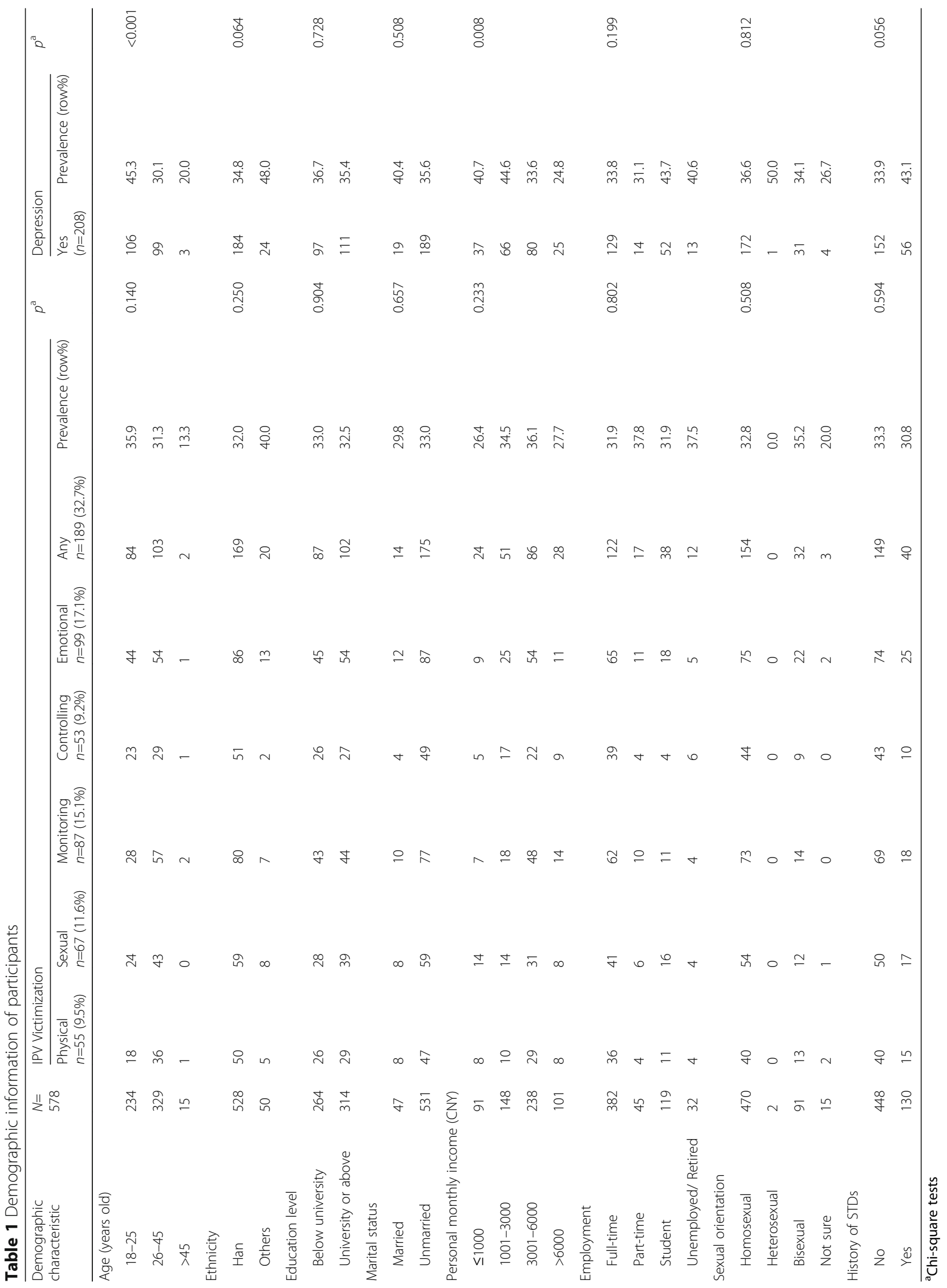


Table 2 Mean, standard deviation of the variable and correlation between variables in the study

\begin{tabular}{|c|c|c|c|c|c|c|c|}
\hline Variables & $\begin{array}{l}\text { IPV } \\
\text { Victimization }\end{array}$ & $\begin{array}{l}\text { Self- } \\
\text { efficacy }\end{array}$ & Depression & $\begin{array}{l}\text { Self- } \\
\text { stigma }\end{array}$ & Mean & SD & Range \\
\hline IPV Victimization & - & & & & 0.62 & 1.11 & $0-5$ \\
\hline Self-efficacy & $-.217^{* *}$ & - & & & 27.45 & 6.00 & $10-40$ \\
\hline Depression & $.206^{* *}$ & $-.536^{* *}$ & - & & 7.55 & 5.75 & $0-30$ \\
\hline Self-stigma & $.118^{* *}$ & $-.363^{* *}$ & $.317^{* *}$ & - & 1.94 & 0.59 & $1-4$ \\
\hline
\end{tabular}

\section{Mediation analysis by SEM Measurement model}

As illustrated in Table 3, all of the standardized factor loading of the measurement model were all statistically significant at the level of $p<0.001$, which ranged from 0.743 to 0.948 . The test of the measurement model resulted in the following statistical values: $\mathrm{X}^{2} / \mathrm{df}$ ratio $=$ 4.29, $\mathrm{CFI}=0.96, \quad \mathrm{IFI}=0.96, \mathrm{RMSEA}=0.075(90 \% \mathrm{CI}=$ $0.064,0.087)$. With inspection of modification indices (MI), correlation path between self-efficacy parcels residuals (between "parcel 1e" and "parcel 3e") and depression parcels residuals (between "parcel $2 \mathrm{~d}$ " and "parcel $3 d$ ") with the largest MI values were added. The modified measurement model yielded a satisfactory model fit: $\chi^{2} / \mathrm{df}$ ratio $=2.91, \mathrm{CFI}=0.98, \mathrm{IFI}=0.98, \mathrm{RMSEA}=0.058$ (90\% CI $=0.046,0.070)$.

\section{Structural model}

Three models representing different versions of potential mediation roles of self-stigma and self-efficacy between IPV and depression are depicted in Fig. 1. Model 1 was a parallel mediation model between IPV and depression, including the direct path and indirect paths via selfstigma and self-efficacy. Model 2 (to Model 1) was a fully indirect set of paths from IPV to depression via self-stigma and self-efficacy. This model excluded the

Table 3 Unstandardized and standardized loading for measurement model

\begin{tabular}{lll}
\hline Parameter estimate & $\begin{array}{l}\text { Unstandardized } \\
\text { Estimate }\end{array}$ & $\begin{array}{l}\text { Standardized } \\
\text { Estimate }\end{array}$ \\
\hline stigma $\rightarrow$ Affective & 1 & 0.902 \\
stigma $\rightarrow$ Cognitive & $0.975^{* * *}$ & 0.948 \\
stigma $\rightarrow$ Behavioral & $0.698^{* * *}$ & 0.767 \\
efficacy $\rightarrow$ Parcel 1e & 1 & 0.866 \\
efficacy $\rightarrow$ Parcel 2e & $1.103^{* * *}$ & 0.905 \\
efficacy $\rightarrow$ Parcel 3e & $1.487^{* * *}$ & 0.859 \\
depression $\rightarrow$ Parcel 1d & 1 & 0.796 \\
depression $\rightarrow$ Parcel 2d & $1.254^{* * *}$ & 0.820 \\
depression $\rightarrow$ Parcel 3d & $1.008^{* * *}$ & 0.743 \\
\hline
\end{tabular}

${ }^{a}$ Items of General Self-Efficacy scale and Center for Epidemiologic Studies Depression 10 were randomly divided into three parcels *** $p<0.001$ direct effect to see if it fits the data better. Model 3 (to Model 1) added a path from self-stigma to self-efficacy. This was designed to check whether some of the association between self-stigma and depression were indirect.

Results of these three model tests are presented in Table 4 . Model 1 explained $43.7 \%$ of the variance in depression but the model fit was unsatisfactory $\left[\mathrm{X}^{2} /\right.$ $\mathrm{df}$ ratio $=4.31, \mathrm{CFI}=0.96, \mathrm{IFI}=0.96, \quad \mathrm{RMSEA}=0.076$ (90\% CI $0.065,0.087)]$. The direct path from IPV victimization to depression was not statistically significant.

Model 2 accounted for $43.5 \%$ of the variance in depression and the model fit did not have significant improvement by excluding the direct path from IPV to depression $(p=0.527$, Table 4$)$. The association between IPV victimization and depression was fully mediated by homosexual self-stigma and self-efficacy. Model 2 yielded an unsatisfactory fit $\left[\chi^{2} / \mathrm{df}\right.$ ratio $=4.23, \mathrm{CFI}=$ 0.96, IFI $=0.96$, RMSEA $=0.075$ (90\% CI 0.064, 0.086) . The standardized path coefficients of Model 2 are also presented in Fig. 1.

The fit of Model 3 improved significantly as compared to Model $1(p<0.001$, Table 4$)$. Homosexual self-stigma was significantly associated with self-efficacy. Model 3 yielded a satisfactory fit $\left[\chi^{2} / \mathrm{df}\right.$ ratio $=2.75, \mathrm{CFI}=0.98$, IFI $=0.98, \quad$ RMSEA $=0.055(90 \%$ CI $0.044,0.067)]$; the overall Model 3 explained $47.8 \%$ of the variance in depression. All standardized path coefficients of Model 3 were statistically significant (range: 0.14 to -0.58 , all $p<0.001$ ) except for the direct path from IPV to depression.

Model 3 was thus selected as the final model. Bootstrapping was used to estimate the indirect effect of self-stigma and self-efficacy. Three significant indirect paths from IPV and depression were found, 1) through self-stigma, the indirect effect was 0.031 (95\% CI: $0.008,0.069) ; 2)$ through self-efficacy, the indirect effect was 0.084 (95\% CI: 0.032, 0.145); and 3) through self-stigma and self-efficacy, the indirect effect was 0.033 (95\% CI: 0.020, 0.034) (Table 5).

\section{Discussion}

The study examined the association between IPV victimization and depressive symptoms among Chinese MSM and tested the mediation roles of homosexual self- 


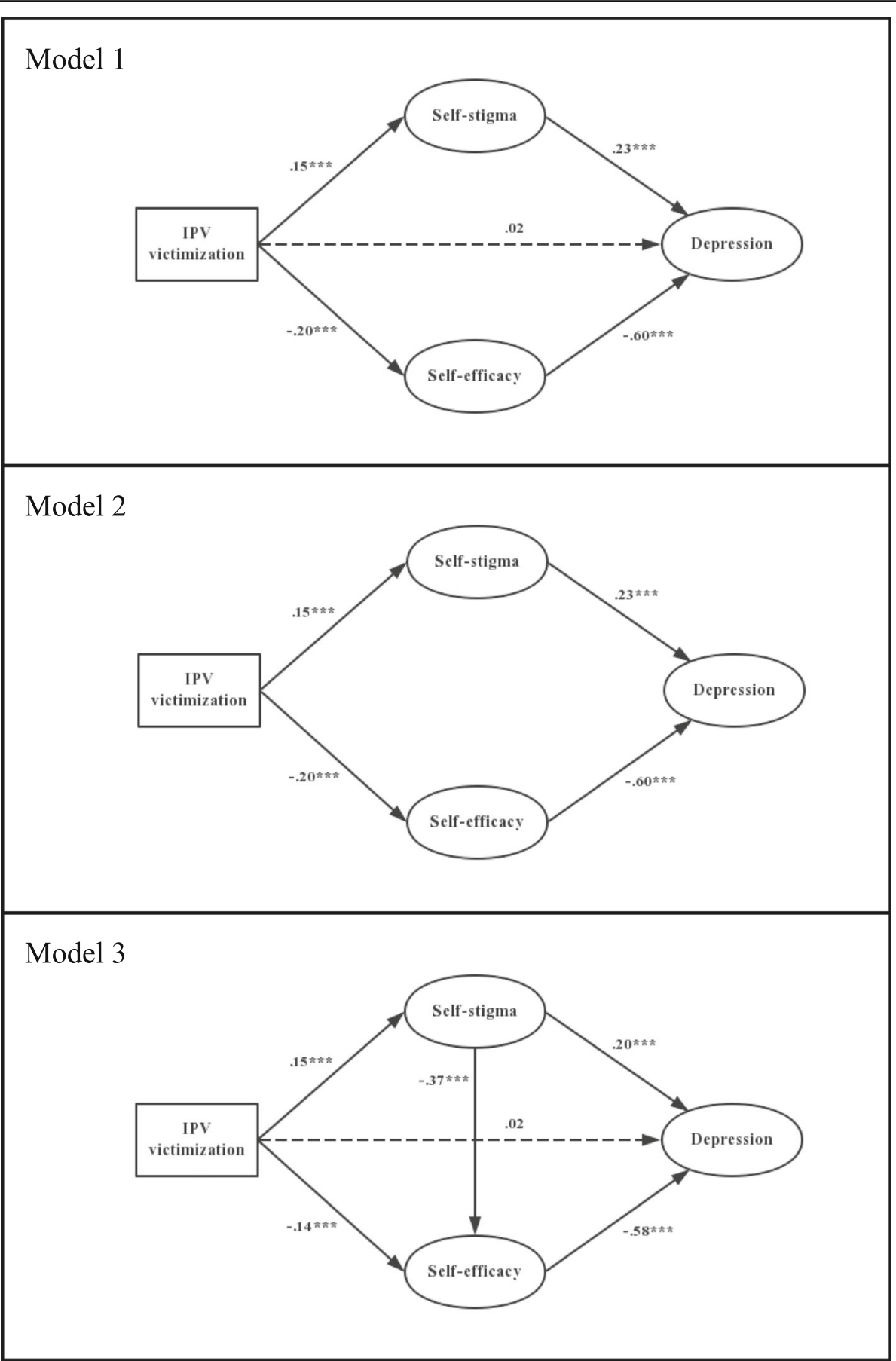

Fig. 1 Hypothesized structural models (Model 1,2 and 3). Note: All path coefficients shown were standardized. ${ }^{* * *} p<0.001$. Age and income have been controlled for in all SEM analyses and were not drawn in the figure

stigma and self-efficacy. The findings showed that prevalences of lifetime IPV victimization and probable depression were high among Chinese MSM. The mediation model further revealed the association between level of IPV victimization and depression was fully mediated via higher homosexual self-stigma and lower self-efficacy.
The prevalence of IPV victimization was 32.7\% (189/ 578), which was higher than the prevalence reported by two prior surveys among MSM in China (29.8 and 24.3\%) [5, 7]. Different from previous studies, the present study included only eligible participants who had or had ever intimate partners, and used a more comprehensive assessment of IPV that involved five 
Table 4 Goodness-of-fit of hypothesized structural model

\begin{tabular}{lllllll}
\hline Models & CFI & IFI & RMSEA & X2/df & X2 & df \\
Model 1 & 0.96 & 0.96 & 0.076 & 4.31 & 198.43 & 46 \\
Model 2 & 0.96 & 0.96 & 0.075 & 4.23 & 198.83 & 47 \\
Model 3 & 0.98 & 0.98 & 0.055 & 2.75 & 123.80 & 45 \\
& & & Changes in X2 & Changes in df & $p$ value \\
Comparisons & & & & \\
Model 2 versus model 1 & 0.40 & & 1 & & 0.527 \\
Model 3 versus model 1 & 74.63 & & 1 & $<0.001$ \\
\hline
\end{tabular}

different domains. Thus the result could be partially attributed to the cross-study variations in methodology (e.g., sample characteristics, definition and measurement of IPV). We also found a prevalence of 36.0\% of depression in the MSM sample, which is comparable to the prevalence reported in previous studies among MSM in China [40, 41]. Younger age and lower income level were associated with increased risk of depression, corroborating with prior studies among Chinese MSM [42, 43]. MSM commonly suffer from stress or rejection due to their sexual identity from family [44]. A qualitative study also showed that internalized homophobia was higher when the MSM population were younger and concealment strategies are often used by young MSM instead of coming out to avoid discrimination and violence associated with stigmatized identity [45]. A study showed that Younger MSM had a higher prevalence of anxiety than older MSM [41], which was also strongly linked to depression. In addition, young MSM were more likely to have sexual risk behaviors and have lower utilization of mental health care, which may further aggravate their psychological problem [46]. Personal income is also associated with depression, which might be partially explained by the financial hardship, discrimination and social stress related to poverty [47]. Mental health interventions and services therefore should be prioritized for younger and poorer MSM, who might be more vulnerable and lack of resources to cope with mental distress.
Consistent with previous studies [48, 49], higher level of IPV victimization was associated with increased risk of depression. The findings further revealed that selfstigma and self-efficacy fully mediated the association between level of IPV and depression. This indicated that IPV experiences increased internalized stigma towards self and was detrimental to self-efficacy, which in turn greatly increased the risk of depression, and psychological interventions to manage negative emotions about one's sexual minority identity and disrupt negative cognitive appraisals may be helpful to prevent depression in MSM who were IPV victims. Our results provided empirical support for the minority stress model, which posits that self-stigma might be induced by external negative experiences and is an important factor in the development of psychopathology among sexual minorities.

In addition, higher level of IPV and self-stigma was associated with lower self-efficacy and for the first time, self-efficacy was confirmed as a mediator in the association between IPV and depression among MSM population. It seemed that, the violent treatment by intimate partners and negative attitudes towards self could be particularly harmful to the personal sense of competence or confidence in managing problems. These findings corroborate a previous study that supported a similar serial mediation model of self-stigma and self-efficacy in the association between prejudiced events and physical health among sexual minority populations [50]. In fact, several studies have suggested that general self-efficacy may be a source of resilience for people involved in aggressive intimate relationships [51]. Thus the results underscored the importance to increase self-efficacy, for example via personal empowerment and cognitivebehavior therapy, in order to reduce the negative impacts of IPV and stigma on mental health in counseling interventions and psycho-educational programming targeted at MSM.

The study has several limitations. First, given the cross-sectional nature of the present study, we cannot infer any conclusive causal relationships which demands longitudinal studies to further reveal the causalities

Table 5 Summary of total, direct, and indirect effects of the mediation model

\begin{tabular}{lll}
\hline Mediation model & Effect $(95 \% \mathrm{Cl})$ & $p$ value \\
\hline Direct effect & & 0.512 \\
$\quad I P V \rightarrow$ depression & $0.024(-0.049,0.103)$ & $<0.001$ \\
Indirect effect & $0.148(0.096,0.199)$ & $<0.001$ \\
$I P V$ victimization $\rightarrow$ self-stigma $\rightarrow$ depression & $0.031(0.008,0.069)$ & $<0.001$ \\
$I P V$ victimization $\rightarrow$ self-efficacy $\rightarrow$ depression & $0.084(0.032,0.145)$ & $<0.001$ \\
$I P V$ victimization $\rightarrow$ self-stigma $\rightarrow$ self-efficacy $\rightarrow$ depression & $0.033(0.020,0.034)$ & $<0.001$ \\
Total effect & $0.172(0.081,0.266)$ & \\
\hline
\end{tabular}


between these variables. Second, the results may not be generalized to other cultures and populations. Third, reporting bias may exist due to the nature of self-reported data. Fourth, although we recruited participants from 15 cities, selection bias may exist as participants were recruited from local gay-friendly organizations using convenience sampling. Local community organizations could be an importance source of social support and thus participants who had close ties to the organization may have better mental health than those who did not. In addition, majority of the study participants were urban residents and may not be representative of general MSM population. Fifth, IPV-GBM used in the present study has not yet been validated in Chinese MSM and further research is warranted to explore the application and psychometric properties in Chinese population.

\section{Conclusion}

In sum, this study is among one of the first studies to examine the mediation role of homosexual self-stigma and self-efficacy between IPV victimization and depression among MSM in China. Both IPV victimization and depression prevalence rates were high among Chinese MSM, which warranted tailored health interventions and services for MSM. The association between IPV victimization and depression was fully mediated by higher homosexual self-stigma and lower self-efficacy. Homosexual self-stigma had a direct effect and an indirect effect via self-efficacy on depression. The results provided implications that integrated interventions that eliminate self-stigma and foster self-efficacy could be promising approaches to reduce depression among MSM with IPV victimization.

\section{Abbreviations \\ CES-D-10: The Center for Epidemiologic Studies Depression 10; CFA: Confirmatory factor analysis; CFI: Comparative fit index; Cl: Confidence interval; GSE: General Self-Efficacy; IFI: Incremental fit index; IPV: Intimate partner violence; IPV-GBM: Intimate partner violence among gay and bisexual men scale; MSM: Men who have sex with men; RMSEA: Root-mean-square error of approximation; SEM: Structural equation modeling; SSS-S: The Self- Stigma Scale-Short Form; STDs: Sexually transmitted diseases}

\section{Acknowledgments}

We thank the local NGO, Chengdu Tongle Health Counseling Service Center for its support. We would like to thank all participants who have taken part in this study and all field workers for their support on data collection.

\section{Authors' contributions}

$J \mathrm{~L}$ and RS have coordinated and designed the project. LP, JL, DW and FH have selected the psychological scales. JL, RS, JG, CH and FH developed and adapted the questionnaire used in the project. JL, LP, JG and $\mathrm{CH}$ have coordinated the fieldwork. LP and DW have organized the fieldwork and data collection. LP, RS and DW have analyzed the data. LP, JL and RS have prepared the first draft of the article. All authors have participated in the edition and revision of the manuscript. All authors have read and approved the final version submitted.

\section{Funding}

This research was supported by the National Natural Science Foundation of China $(81803334,71774178,71974212)$, a Major Infectious Disease Prevention and Control of the National Science and Technology Major Project of China (2018ZX10715004), Science and Technology Planning Project of Guangdong Province (2017A020212006), and Science and Technology Research Project of Guangzhou (201607010332, 201607010368). The funder had no role in study design, data collection, data analysis, data interpretation, or writing the manuscript.

\section{Availability of data and materials}

The datasets used in the study are available from the corresponding author on reasonable request.

\section{Ethics approval and consent to participate}

Ethics approval was obtained from the ethics committee of Sun Yat-sen University (Approval No. [2018] 049). Informed consents were obtained online from the eligible participants before self-administering the questionnaire by clicking a box ("I agree to participate in this study") of the online questionnaire.

\section{Consent for publication}

Not applicable.

\section{Competing interests}

The authors declare that they have no competing interests.

\section{Author details}

${ }^{1}$ School of Public Health, Sun Yat-sen University, No.74, Zhongshan second road, Guangzhou, China. ${ }^{2}$ Centre for Health Behaviours Research, JC School of Public Health and Primary Care, The Chinese University of Hong Kong, Hong Kong, SAR, China. ${ }^{3}$ Sun Yat-sen Global Health Institute, Sun Yat-sen University, Guangzhou, China. ${ }^{4}$ Department of Public Mental Health,

Shenzhen Kangning Hospital, Shenzhen, Guangdong, China.

Received: 23 September 2019 Accepted: 24 December 2019

Published online: 03 January 2020

\section{References}

1. Finneran C, Stephenson R. Intimate partner violence among men who have sex with men: a systematic review. Trauma Violence Abus. 2013;14(2):168-85.

2. Intimate partner violence: Definitions [https://www.cdc.gov/ violenceprevention/intimatepartnerviolence/definitions.html]. 15 Aug 2019.

3. Buller AM, Devries KM, Howard LM, Bacchus L. Associations between intimate partner violence and health among men who have sex with men: a systematic review and meta-analysis. PLoS Med. 2014;11(3):e1001609.

4. Dunkle KL, Wong FY, Nehl EJ, Lin L, He N, Huang J, Zheng T. Male-on-male intimate partner violence and sexual risk behaviors among money boys and other men who have sex with men in Shanghai, China. Sex Transm Dis. 2013;40(5):362-5.

5. Liu $Y$, Zhang $Y$, Ning Z, Zheng H, Ding $Y$, Gao M, Wong FY, He N. Intimate partner violence victimization and HIV infection among men who have sex with men in Shanghai, China. Biosci Trends. 2018;12(2):142-8.

6. Wang HY, Wang N, Chu ZX, Zhang J, Mao X, Geng WQ, Jiang YJ, Shang H, $\mathrm{Xu} \mathrm{JJ}$. Intimate partner violence correlates with a higher HIV incidence among MSM: a 12-month prospective cohort study in Shenyang, China. Sci Rep. 2018;8(1):2879.

7. Davis A, Best J, Wei C, Luo J, Van Der Pol B, Meyerson B, Dodge B, Aalsma M, Tucker J, Social Entrepreneurship for Sexual Health Research G. Intimate partner violence and correlates with risk behaviors and HIV/STI diagnoses among men who have sex with men and men who have sex with men and women in China: a hidden epidemic. Sex Transm Dis. 2015;42(7):387-92.

8. Miltz AR, Lampe FC, Bacchus LJ, McCormack S, Dunn D, White E, Rodger A, Phillips AN, Sherr L, Clarke A, et al. Intimate partner violence, depression, and sexual behaviour among gay, bisexual and other men who have sex with men in the PROUD trial. BMC Public Health. 2019;19(1):431.

9. Stephenson R, Finneran C. Minority stress and intimate partner violence among gay and bisexual men in Atlanta. Am J Mens Health. 2017;11(4): 952-61. 
10. Meyer $\mathrm{IH}$. Minority stress and mental health in gay men. J Health Soc Behav. 1995;36(1):38-56.

11. Meyer $H$. Prejudice, social stress, and mental health in lesbian, gay, and bisexual populations: conceptual issues and research evidence. Psychol Bull. 2003;129(5):674-97.

12. Yan H, Li XY, Li JJ, Wang W, Yang YM, Yao X, Yang NX, Li SY. Association between perceived HIV stigma, social support, resilience, self-esteem, and depressive symptoms among HIV-positive men who have sex with men (MSM) in Nanjing, China. Aids Care. 2019;31(9):1069-76.

13. Tao J, Wang L, Kipp AM, Qian HZ, Yin L, Ruan YH, Shao YM, Lu HY, Vermund $\mathrm{SH}$. Relationship of stigma and depression among newly HIV-diagnosed Chinese men who have sex with men. AIDS Behav. 2017;21(1):292-9.

14. Li Z, Hsieh E, Morano JP, Sheng Y. Exploring HIV-related stigma among HIVinfected men who have sex with men in Beijing, China: a correlation study. AIDS Care. 2016;28(11):1394-401.

15. Hatzenbuehler ML. How does sexual minority stigma "get under the skin"? A Psychological Mediation Framework. Psychol Bull. 2009;135(5):707-30.

16. Brewster ME, Moradi B, DeBlaere C, Velez BL. Navigating the borderlands: the roles of minority stressors, bicultural self-efficacy, and cognitive flexibility in the mental health of bisexual individuals. J Couns Psychol. 2013;60(4):543-56.

17. Zhang LY, Li XM, Wang B, Shen ZY, Zhou YJ, Xu JP, Tang ZZ, Stanton B. Violence, stigma and mental health among female sex workers in China: a structural equation modeling. Women Health. 2017;57(6):685-704.

18. Bandura A. Self-efficacy: toward a unifying theory of behavioral change. Psychol Rev. 1977:84(2):191-215.

19. Bandura A. The Explanatory and Predictive Scope of Self-Efficacy Theory. J Soc Clin Psychol. 1986;4:359-73.

20. Swan $\mathrm{H}, \mathrm{O}^{\prime}$ Connell DJ. The impact of intimate partner violence on women's condom negotiation efficacy. J Interpers Violence. 2012;27(4):775-92.

21. Neilson EC, Gilmore AK, Stappenbeck CA, Gulati NK, Neilon E, George WH, Davis KC. Psychological effects of abuse, partner pressure, and alcohol: the roles of in-the-moment condom negotiation efficacy and condom-decision abdication on Women's intentions to engage in Condomless sex. J Interpers Violence. 2019;886260519857160. https://journals.sagepub.com/ doi/full/10.1177/0886260519857160.

22. Calvete E, Estevez A, Corral S. Intimate partner violence and depressive symptoms in women: cognitive schemas as moderators and mediators. Behav Res Ther. 2007:45(4):791-804.

23. Wang N, Wang S, Qian HZ, Ruan Y, Amico KR, Vermund SH, Yin L, Qiu X, Zheng S. Negative associations between general self-efficacy and anxiety/ depression among newly HIV-diagnosed men who have sex with men in Beijing, China. Aids Care. 2019;31(5):629-35.

24. Maciejewski PK, Prigerson HG, Mazure CM. Self-efficacy as a mediator between stressful life events and depressive symptoms. Differences based on history of prior depression. Br J Psychiatry. 2000;176:373-8.

25. Molina Y, Ramirez-Valles J. HIV/AIDS stigma: measurement and relationships to psycho-behavioral factors in Latino gay/bisexual men and transgender women. AIDS Care. 2013;25(12):1559-68

26. Seghatol-Eslami VC, Dark HE, Raper JL, Mugavero MJ, Turan JM, Turan B. Brief Report: Interpersonal and Intrapersonal Factors as Parallel Independent Mediators in the Association Between Internalized HIV Stigma and ART Adherence. J Acquir Immune Defic Syndr. 2017;74(1):e18-22.

27. Zhou G, Li X, Qiao S, Shen Z, Zhou Y. HIV symptom management selfefficacy mediates the relationship of internalized stigma and quality of life among people living with HIV in China. J Health Psychol. 2017; 1359105317715077. https://journals.sagepub.com/doi/full/10.1177/13591 05317715077

28. Stephenson R, Finneran C. The IPV-GBM scale: a new scale to measure intimate partner violence among gay and bisexual men. PLoS One. 2013;8(6):e62592.

29. Li J, Mo PK, Wu AM, Lau JT. Roles of self-stigma, social support, and positive and negative affects as determinants of depressive symptoms among HIV infected men who have sex with men in China. AIDS Behav. 2017;21(1):261-73.

30. Han Y, Xia D, Sun Y, Li G, Lu H, He X, Li X, Shao Y, Ruan Y. HIV prevalence and its related factors among men who have sex with men in Beijing. Chin J AIDS STD. 2013;19(06):399-401+412.

31. Qiu X, Zhang J, Xie A, Ye H, Li S, Gong W, Jiang G, Cui D, Qi X, Tao Y, et al. Study on the effect of general self-efficacy on knowledge and behavior about AIDS in MSM. Pract Prev Med. 2013;20(11):1297-300.

32. Li D, Li C, Wang Z, Lau JTF. Prevalence and associated factors of unprotected anal intercourse with regular male sex partners among HIV negative men who have sex with men in China: a cross-sectional survey. PLoS One. 2015;10(3):e0119977.

33. Wei C, Cheung DH, Yan H, Li J, Shi L-E, Raymond HF. The Impact of Homophobia and HIV Stigma on HIV Testing Uptake Among Chinese Men Who Have Sex With Men: a Mediation Analysis. J Acquir Immune Defic Syndr. 2016;71(1):87-93.

34. Su X, Zhou AN, Li J, Shi L-E, Huan X, Yan H, Wei C. Depression, Loneliness, and Sexual Risk-Taking Among HIV-Negative/Unknown Men Who Have Sex with Men in China. Arch Sex Behav. 2018;47(7):1959-68.

35. Miller WC, Anton HA, Townson AF. Measurement properties of the CESD scale among individuals with spinal cord injury. Spinal Cord. 2008;46(4):287-92.

36. Kline RB. Principles And Practice Of Structural Equation Modeling; 2010.

37. Hopper D, Coughlan J, Mullen MR: Structural equation modeling: Guidelines for determining model fit. The Electronic Journal of Business Research Methods. 2008;6:53-60.

38. Browne MW, Cudeck R. Alternative Ways of Assessing Model Fit. Sociol Methods Res. 1992;21. https://journals.sagepub.com/doi/10.1177/00491241 92021002005

39. Preacher KJ, Hayes AF. Asymptotic and resampling strategies for assessing and comparing indirect effects in multiple mediator models. Behav Res Methods. 2008;40(3):879-91.

40. Yu L, Li Y, Liu L, Li S, Na J, An X, Zhou Y, Gu Y, Bi X, Mu H et al. Association of recent gay-related stressful events and emotional distress with suicidal behaviors over 12 months in Chinese men who have sex with men. AsiaPac Psychiat. 2018;10(1):e12286.

41. Hu Y, Zhong XN, Peng B, Zhang Y, Liang H, Dai JH, Zhang J, Zhong XH, Huang AL. Comparison of depression and anxiety between HIV-negative men who have sex with men and women (MSMW) and men who have sex with men only (MSMO): a cross-sectional study in Western China. BMJ Open. 2019;9(1):e023498.

42. Liu J, Gao YH, Liang ZM, Li Y, Yang Y. Depressive symptoms and associated sexual behaviors among men who have sex with men in Foshan, Guangdong province. Chin J Epidemiol. 2012;33(5):483-7.

43. Jiang $H$, Chen $X$, Li J, Tan Z, Cheng W, Yang Y. Predictors of condom use behavior among men who have sex with men in China using a modified information-motivation-behavioral skills (IMB) model. BMC Public Health. 2019;19(1):261.

44. Hidalgo MA, Cotten C, Johnson AK, Kuhns LM, Garofalo R. 'Yes, I am more than just that': gay/bisexual young men residing in the United States discuss the influence of minority stress on their sexual risk behavior prior to Hiv infection. Int J Sex Health. 2013;25(4):291-304.

45. McAdams-Mahmoud A, Stephenson R, Rentsch C, Cooper H, Arriola K, Jobson G, de Swardt G, Struthers H, McIntyre J. Minority stress in the lives of men who have sex with men in Cape Town, South Africa. J Homosex. 2014;61(6):847-67.

46. Salomon EA, Mimiaga MJ, Husnik MJ, Welles SL, Manseau MW, Montenegro AB, Safren SA, Koblin BA, Chesney MA, Mayer KH. Depressive Symptoms, Utilization of Mental Health Care, Substance Use and Sexual Risk Among Young Men Who have Sex with Men in EXPLORE: Implications for AgeSpecific Interventions. Aids Behav. 2009;13(4):811-21.

47. Duncan DT, Park SH, Schneider JA, Al-Ajlouni YA, Goedel WC, Elbel B, Morganstein JG, Ransome Y, Mayer KHJA. Financial hardship, Condomless Anal Intercourse and HIV Risk Among Men Who Have Sex with Men. AIDS Behav. 2017;21(12):3478-85.

48. Roesch PT, Velonis AJ, Sant SM, Habermann LE, Hirschtick JL. Implications of interpersonal violence on population mental health status in a low-income Urban Community-based sample of adults. J Interpers Violence. 2019:886260519862365. https://journals.sagepub.com/doi/full/10.1177/0886260519862365.

49. Oh SS, Kim W, Jang SI, Park EC. The association between intimate partner violence onset and gender-specific depression: a longitudinal study of a nationally representative sample. J Affect Disord. 2019;250:79-84.

50. Denton F, Rostoksy SS, Danner F. Minority stress, coping self-efficacy, and physical health in lesbians, gays, and bisexuals; 2014.

51. Sullivan TP, MCPartland T, Price C, Cruza-Guet MC, Swan SC. Relationship self-efficacy protects against mental health problems among women in bidirectionally aggressive intimate relationships with men. J Couns Psychol. 2013;60(4):641-7.

\section{Publisher's Note}

Springer Nature remains neutral with regard to jurisdictional claims in published maps and institutional affiliations. 